\title{
A Comparative Study of Modal Properties of Surface-Emitting Circular Bragg Micro-Lasers
}

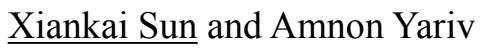 \\ Department of Applied Physics, California Institute of Technology, 1200 E. California Blvd., Pasadena, CA 91125, USA \\ E-mail: xksun@,caltech.edu
}

\begin{abstract}
Modal properties of two types of surface-emitting circular Bragg micro-lasers (CBMLs) are compared. Disk CBMLs are suitable for low-threshold, high-efficiency, ultracompact laser design, while ring CBMLs are an excellent candiadate for high-efficiency, large-area, high-power applications.
\end{abstract}

Keywords: Surface-emitting lasers; Semiconductor lasers; Bragg lasers; Modal property; Integrated optoelectronics

\section{INTRODUCTION}

Surface-emitting lasers with a circular emission aperture are particularly useful in optical communications because they can produce circularly-symmetric, narrow-divergence laser beams [1-3]. With optical gain, circular Bragg microresonators can be engineered for such applications - the radial Bragg reflector not only provides feedback for the in-plane fields but also couples the laser radiation out of the plane vertically. The lasers based on such circular Bragg resonance are referred to as circular Bragg micro-lasers (CBMLs).

Similar to the circular resonators, the CBMLs can be classified into two configurations: disk- and ring- CBMLs. As shown in Fig. 1, disk CBML has a center disk surrounded by a radial Bragg grating extending from $x_{0}$ to $x_{b}$. Ring CBML has an annular defect surrounded by inner and outer gratings which extends, respectively, from the center to $x_{L}$ and from $x_{R}$ to $x_{b}$. In this paper we compare their modal properties such as modal field pattern, threshold gain, detuning factor, and far-filed pattern. In order to demonstrate their advantages as surface-emitting lasers, circular DFB laser will be introduced as a reference in comparing the quality factor, emission efficiency, and modal area.

\section{COMPARISON OF MOdAl PROPERTIES OF SURFACE-EMITTING CBMLS}

The characteristic equations which govern the laser modes were derived in [4] by resonance condition theory:

$$
\begin{aligned}
& \frac{e^{2\left(g_{A}-i \delta\right) x_{0}} v \sinh \left[S\left(x_{b}-x_{0}\right)\right]}{(u-i \delta) \sinh \left[S\left(x_{b}-x_{0}\right)\right]-S \cosh \left[S\left(x_{b}-x_{0}\right)\right]}=1 \text { for disk CBML, } \\
& \text { and } \frac{e^{2\left(g_{A}-i \delta\right)\left(x_{R}-x_{L}\right)} v \sinh \left[S\left(x_{b}-x_{R}\right)\right]}{(u-i \delta) \sinh \left[S\left(x_{b}-x_{R}\right)\right]-S \cosh \left[S\left(x_{b}-x_{R}\right)\right]} \\
& \quad \times \frac{(u-v-i \delta) \sinh \left[S x_{L}\right]+S \cosh \left[S x_{L}\right]}{-(u-v-i \delta) \sinh \left[S x_{L}\right]+S \cosh \left[S x_{L}\right]}=1 \text { for ring CBML. }
\end{aligned}
$$
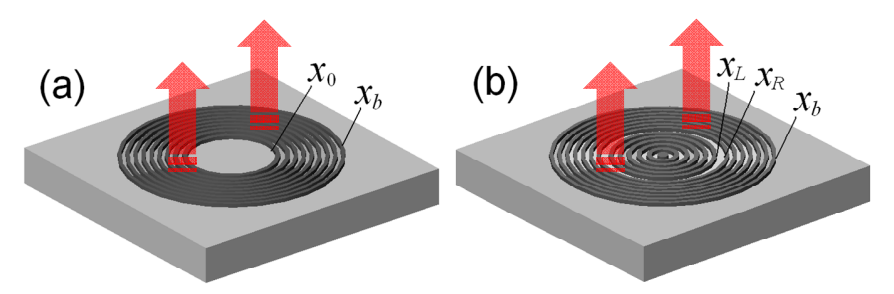

Fig. 1. Illustrations of surface-emitting CBMLs: (a) Disk CBML and (b) Ring CBML. Laser radiation is coupled out of the plane in vertical direction by the first-order scattering.

TABLE I

MODAL FIELD PATTERNS, THRESHOLD GAINS $\left(g_{A}\right.$, IN UNIT OF $\left.10^{-3}\right)$, AND

\begin{tabular}{|c|c|c|c|c|c|c|}
\hline Laser type & \multicolumn{3}{|c|}{ Disk CBML } & \multicolumn{3}{|c|}{ Ring CBML } \\
\hline & Modal field & $g_{A}$ & $\delta$ & Modal field & $g_{A}$ & $\delta$ \\
\hline Mode 1 & Whannow & 0.127 & 49.8 & & 0.457 & 55.9 \\
\hline Mode 2 & WNAWW & 0.288 & 21.2 & & 1.06 & 66.9 \\
\hline Mode 3 & Wham & 0.454 & -8.09 & & 1.92 & 71.0 \\
\hline Mode 4 & Hewawnown & 0.690 & -37.4 & & 3.14 & 84.4 \\
\hline Mode 5 & Lhanumwann & 1.21 & -66.5 & & 4.09 & 91.6 \\
\hline
\end{tabular}
FREQUENCY DETUNING FACTORS $\left(\delta\right.$, IN UNIT OF $\left.10^{-3}\right)$ OF THE DISK CBML AND RING CBML WITH EXTERIOR BOUNDARY RADIUS $x_{b}=200$
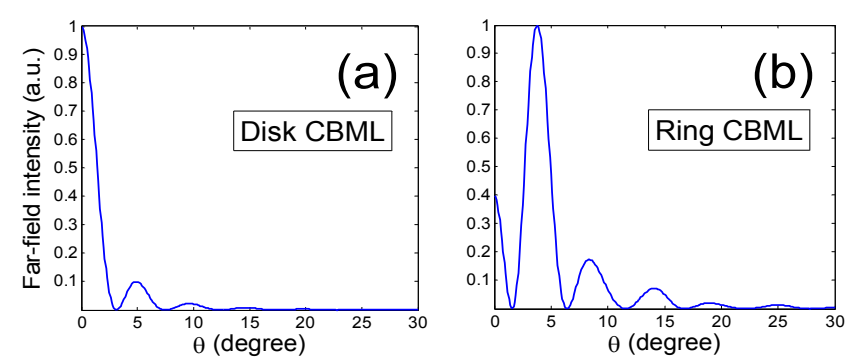

Fig. 2. Far-field intensity patterns of the fundamental modes of disk CBML and ring CBML (both with $x_{b}=200$ ).

In the left equations, $g_{A}$ is the gain coefficient. $\delta=\left(\beta_{\text {design }}-\beta\right) / \beta$ is the frequency detuning factor, where $\beta$ is the in-plane propagation constant. $u$ and $v$ are defined as $u=g_{A}-h_{1}, v=h_{1}$ 

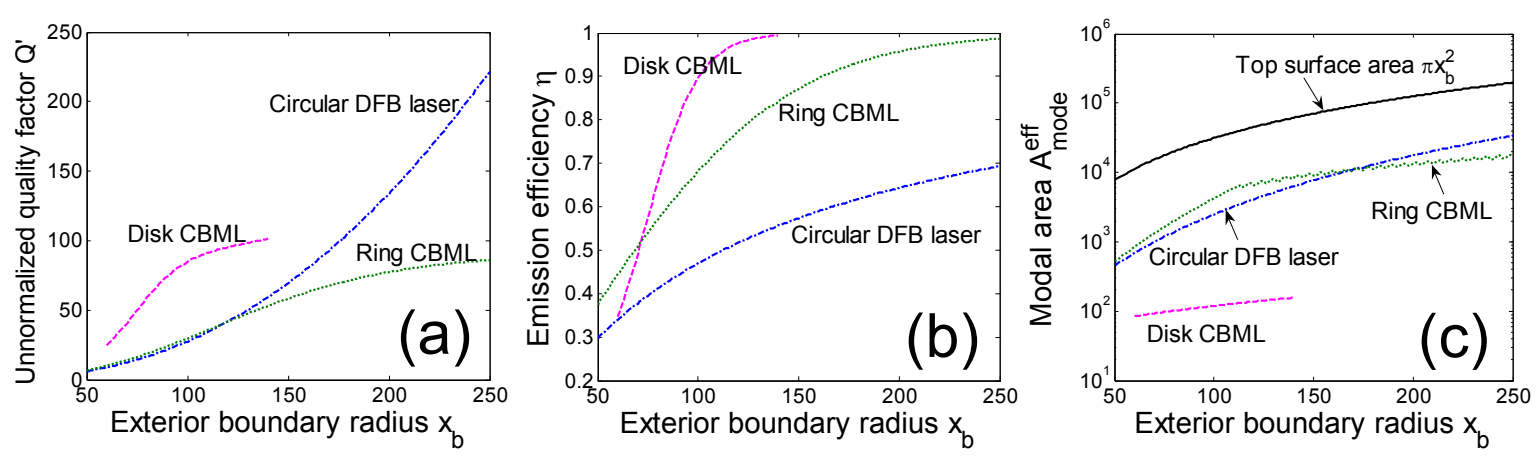

Fig. 3. (a) Unnormalized quality factors, (b) emission efficiencies, and (c) modal areas of the fundamental modes of disk CBMLs (dashed), ring CBMLs (dotted), and circular DFB lasers (dash-dot).

$+i h_{2}$, where $h_{1}$ and $h_{2}$ are the radiation- and feedbackcoupling coefficients which can be precalculated. $S$ is defined as $S=\left[(u-i \delta)^{2}-v^{2}\right]^{1 / 2}$. It should be noted that these characteristic equations implicitly includes the effect of vertical radiation for nonzero $h_{1}$.

Modal field patterns, threshold gains $g_{A}$, and frequency detuning factors $\delta$ of the disk- and ring- CBMLs are listed in Table I for devices with $x_{b}=200$. For disk CBML, the inner disk radius $x_{0}=x_{b} / 2$. For ring CBML, the annular defect is assumed to be located at $x_{b} / 2$ with its width being a wavelength such that $x_{L}+x_{R}=x_{b}$ and $x_{R}-x_{L}=2 \pi$. As shown in Table I, all the displayed modes of disk CBML are confined to the center disk with negligible peripheral power leakage and thus possess very low thresholds and very small modal areas. For ring CBML, each mode needs a higher threshold gain than that of the disk CBML, but they also possess large modal areas which is advantageous for large-aperture, high-power surface emission.

Far-field intensities are calculated based on Fourier transform of the radiation fields at the emission aperture. Shown in Fig. 2, the disk CBML has most of the energy concentrated in the zeroth-order Fourier component thus its peak is located at the center, while the ring CBML has the first-order lobe dominating thus features a donut shape.

In what follows we also include circular DFB laser as a reference to show the advantages of CBMLs as surfaceemitting lasers. To compare quality factor, an unnormalized

$$
Q^{\prime}=\frac{\int_{0}^{D} Z^{2}(z) d z \cdot \int_{0}^{x_{b}}|E(x)|^{2} x d x}{\int_{\text {grating }}|\Delta E(x, z=0)|^{2} x d x+\int_{0}^{D} Z^{2}(z) d z \cdot\left|E\left(x=x_{b}\right)\right|^{2} \beta x_{b}}
$$

is used whose nominator represents the store energy and the denominator the total power loss. $D$ is the resonator thickness and $Z$ is the vertical modal profile. $E(x)$ denotes the in-plane modal field and $\Delta E(x, z)$ the radiation field where the emission aperture is located at $z=0$. Fig. 3(a) shows increases in the device size $\left(x_{b}\right)$ result in enhanced $Q^{\prime}$ values. Additionally, the disk CBML exhibits a much higher $Q^{\prime}$ than the other two laser structures of identical dimensions, which is consistent with the threshold behaviors shown in Table I.

The emission efficiency $\eta$ is naturally defined as the fraction of the total power loss which is represented by the useful vertical radiation. Fig. 3(b) demonstrates a larger $\eta$ for devices with a larger size $\left(x_{b}\right)$. As seen from comparison, only the disk- and ring- CBMLs achieve high emission efficiencies. This is because their fundamental modes are bandgap modes while that of the circular DFB laser is an in-band mode.

The effective modal area in a 2-D case is defined as

$$
A_{\text {mode }}^{\text {eff }}=\left[\iint|\mathbf{E}|^{2} x d x d \varphi\right] / \max \left\{|\mathbf{E}|^{2}\right\},
$$

where $x$ and $\varphi$ are radial and angular coordinates, respectively. It is a measure of how the modal field is distributed in the laser. Plotted in Fig. 3(c), the modal area of the disk CBML was found to be the smallest. This is not surprising and can be inferred from their unique modal profiles listed in Table I. Actually our prior experimental work has demonstrated single-mode lasing with ultrasmall modal volume in a nanosized disk CBML fabricated in InGaAsP [3]. Clearly, a disk configuration is preferable in ultracompact laser design. Alternatively, ring CBMLs have a large modal area with high emission efficiency, rendering them suitable for highefficiency, high-power, large-area laser applications.

\section{CONCLUSIONS}

By comparing the modal properties of disk- and ringCBMLs, we demonstrated their advantages as surfaceemitting lasers and concluded that disk CBMLs are most useful in low-threshold, high-efficiency, ultracompact laser design, while ring CBMLs are excellent candidates for highefficiency, high-power, large-area lasers.

\section{REFERENCES}

[1] T. Erdogan, O. King, G. W. Wicks, D. G. Hall, E. H. Anderson, and M. J. Rooks, "Circularly symmetric operation of a concentric-circle-grating, surface-emitting, AlGaAs/GaAs quantum-well semiconductor laser," Appl. Phys. Lett., vol. 60, pp. 1921-1923, Apr. 1992.

[2] M. Fallahi, M. Dion, F. Chatenoud, I. M. Templeton, K. A. McGreer, G. Champion, and R. Barber, "Low divergence electrically pumped circular-grating surface-emitting DBR laser on an InGaAs/GaAs structure," Electron. Lett., vol. 29, pp. 1412-1414, Aug. 1993.

[3] J. Scheuer, W. M. J. Green, G. A. DeRose, and A. Yariv, "Lasing from a circular Bragg nanocavity with an ultrasmall modal volume," Appl. Phys. Lett., vol. 86, p. 251101, Jun. 2005.

[4] X. K. Sun and A. Yariv, "A unified theory for surface emitting chirped circular grating lasers," in Proc. SPIE. vol. 7218, J. E. Broquin and C. M. Greiner, Eds. SPIE Photonics West 2009, San Jose, CA: SPIE, 2009, p. $72180 \mathrm{H}$. 